\title{
ON SPECIAL JORDAN ALGEBRAS
}

\author{
BY \\ G. K. KALISCH
}

1. Introduction. Let $\subseteq$ be an associative algebra of finite order over any field $\mathfrak{F}$ of characteristic not two and $a b$ represent the product of any two quantities $a$ and $b$ of $\mathfrak{S}$. Then the operation

$$
a \cdot b=(a b+b a) / 2
$$

for $a$ and $b$ in $\subseteq$ is called quasimultiplication, and any linear subspace $\mathfrak{A}$ over $\mathfrak{F}$ of $\mathfrak{S}$ which is closed with respect to this operation forms a corresponding algebra $\mathfrak{A}$. We call an algebra isomorphic to such an algebra a special Jordan algebra and see that special Jordan algebras are commutative but not, in general, associative. In the first three of four papers [6-9]( $\left.{ }^{1}\right)$ Jordan considered the class of all algebras satisfying the property $x\left(y x^{2}\right)=(x y) x^{2}$ for all $x$ and $y$. In the last paper the assumptions of reality and commutativity are made.

It is the purpose of this paper to begin a study of simple special Jordan algebras. Any associative algebra $\subseteq$ forms a special Jordan algebra $\mathfrak{S}^{0}$ with respect to quasimultiplication and we shall show that if $\subseteq$ is simple so is $\mathfrak{S}^{0}$. If $\mathfrak{\subseteq}$ has an involution $J$ and $\mathfrak{S}_{J}$ is the set of all $J$-symmetric quantities of $\mathfrak{\subseteq}$, the set $\widetilde{S}_{J}$ is a special Jordan algebra under quasimultiplication. Then we shall show again that $\mathfrak{S}_{J}$ is simple if $\mathfrak{S}_{\text {is. }}$

Our study is analogous to that which has already been made for Lie algebras by Jacobson and Landherr $[3,4,5,10,11]$, and we shall show, as in that theory, that if $\Omega$ is a scalar extension of $\mathfrak{F}$ such that $\mathfrak{A}_{\Omega}$ is a special Jordan algebra of one of the types above, then $\mathfrak{A}$ is a special Jordan algebra of a corresponding type. The analogous analysis for Lie algebras formed a major part of the determination of all simple Lie algebras over any field of characteristic not two and it is expected that our study will occupy a corresponding place in the theory of Jordan algebras.

Many thanks are due to Professor A. A. Albert for suggesting the topic, and guiding the writing, of the present paper which was accepted as a doctoral thesis at the University of Chicago.

2. Involutions. An involution of an associative algebra $\subseteq$ over $\mathfrak{F}$ is a linear transformation $J$ over $\mathfrak{F}$ of $\mathfrak{S}$ such that $J^{2}=I$, the identity transformation, $(a b)^{J}=b^{J} a^{J}$ for every $a$ and $b$ of $\mathfrak{S}$. The quantities $a=a^{J}$ of $\mathfrak{S}$ are called $J$-symmetric and span a linear subspace $\mathfrak{S}_{J}$ of $\mathfrak{S}$. The quantities $a=-a^{J}$ of $\mathfrak{S}$ are called $J$-skew and span a linear subspace $\mathfrak{S}_{J}^{s}$ of $\mathfrak{S}$ such that $\mathfrak{S}$ is the

Presented to the Society, September 5, 1941; received by the editors June 27, 1946.

(1) Numbers in brackets refer to the bibliography at the end of the paper. 
supplementary sum $\mathfrak{S}=\mathfrak{S}_{J}+\mathfrak{S}_{J}^{s}$. We now state the following lemma.

LEMMA 1. Let $\mathfrak{S}$ be an associative algebra over $\mathfrak{F}, J$ be an involution over $\mathfrak{F}$ of $\mathfrak{S}, \Omega$ be a scalar extension field of $\mathfrak{F}$. Then $J$ can be extended uniquely to an involution over $\Re$ (which we shall also designate by $J$ ) of $\mathfrak{S}_{\Re}$ and

$$
\left(\widetilde{S}_{\Omega}\right)_{J}=\left(\widetilde{S}_{J}\right)_{\Omega} .
$$

The proof is analogous to that of the corresponding statement of Jacobson [3, p. 509].

We further observe that if $J$ and $J_{0}$ are involutions over $\mathfrak{F}$ such that $k^{J}=k^{J_{0}}$ for every $k$ in the centrum of $\subseteq$ then, by Albert [2, p. 155], $J=J_{0} T$ where $T$ is an inner automorphism of $\subseteq$ generated by a regular $J_{0}$-symmetric quantity or a regular $J_{0}$-skew quantity $t$ of $\mathfrak{S}$. We then see that $a=a^{J}$ is in $\mathfrak{S}_{J}$ if and only if $a=a^{J_{0} T}=t a^{J_{0}} t^{-1}, a t=t a^{J_{0}},(a t)^{J_{0}}=a t^{J_{0}}= \pm a t$. Thus $a t$ is in $\mathfrak{S}_{J_{0}}$ or $\mathfrak{S}_{\mathbf{J}_{0}}^{s}$, respectively. We therefore have the following lemma.

Lemma 2. The set $\mathfrak{S}_{J}=\mathfrak{S}_{J_{0}} t^{-1}$ or $\mathfrak{S}_{J}=\mathfrak{S}_{J_{0}}^{s} t^{-1}$ according as $t$ is $J_{0}$-symmetric or $J_{0}$-skew.

3. Enveloping algebras of subspaces. If $\mathfrak{B}$ is any subset of an algebra $\mathfrak{S}$ over $\mathfrak{F}$ we define its closure $\mathfrak{B}^{*}$ to be the intersection of all subalgebras of $\mathfrak{S}$ containing $\mathfrak{B}$. Thus $\mathfrak{B}^{*}$ is the enveloping subalgebra of $\subseteq$ consisting of all finite polynomials over $\mathfrak{F}$ in the quantities of $\mathfrak{B}$.

If $\mathfrak{A}$ is any linear subspace of an associative algebra $\mathfrak{S}$ and $T$ is an automorphism $a \rightarrow a^{T}$ of $\subseteq$ the linear space $\mathfrak{A}^{T}$ of all $a^{T}$ for $a$ in $\mathfrak{A}$ has the same order as $\mathfrak{A}$, and if $\mathfrak{A}$ is a special Jordan algebra $\mathfrak{A}^{T}$ is clearly a special Jordan algebra isomorphic to $\mathfrak{A}$. The enveloping algebras $\mathfrak{A}^{*}$ and $\left(\mathfrak{A}^{T}\right)^{*}$ are isomorphic associative algebras and if $\mathfrak{A}^{*}=\mathfrak{S}$ then $\left(\mathfrak{U}^{T}\right)^{*}=\mathfrak{S}$. If $\mathfrak{A}=\mathfrak{S}_{J}$ and $K$ is an involution cogredient to $J$ then $K=T^{-1} J T$ for an automorphism $T$ of $\subseteq$ and if $a=a^{J}$ the quantity $\left(a^{T}\right)^{K}=a^{T T^{-1} J T}=a^{T}$. Hence $\mathfrak{S}_{K}=\left(\widetilde{S}_{J}\right)^{T}$. Thus we have:

Lemma 3. If $J$ and $K$ are cogredient the corresponding special Jordan algebras $\mathfrak{S}_{J}$ and $\mathfrak{S}_{K}$ are isomorphic and their enveloping associative algebras $\left(\mathfrak{S}_{J}\right)^{*}$ and $\left(\Im_{K}\right)^{*}$ are isomorphic.

We now consider a special case and prove the following lemma.

LEMMA 4. Let $J$ be the transformation of transposition on a total matric algebra $\mathfrak{S}$ of degree $n$. Then $\left(\mathfrak{S}_{J}\right)^{*}=\mathfrak{S}$.

If $n=1$ we have $\mathfrak{S}_{J}=S_{S}=\left(\mathfrak{S}_{J}\right)^{*}$. Let $n>1$ and $e_{11}, \cdots, e_{i j}, \cdots, e_{n n}$ be an ordinary matric basis of $\mathfrak{S}$ and thus $e_{i j}^{J}=e_{j i}$. Then every $e_{i i}$ and $e_{i j}+e_{j i}$ is in $\mathfrak{S}_{J}, e_{i i}\left(e_{i j}+e_{j i}\right)=e_{i j}$ is in $\left(\mathfrak{S}_{J}\right)^{*}$, for every $i \neq j$. Hence $\left(\mathfrak{S}_{J}\right)^{*}$ contains our basis of $\mathfrak{S}_{\text {and }}\left(\mathfrak{S}_{J}\right)^{*}=\mathfrak{S}$.

Our next special case will be that of a total matric algebra $\subseteq$ of degree $n=2 m$ over $\mathfrak{F}$. Then $\mathfrak{S}=\mathfrak{M} \times \mathfrak{N}$ where $\mathfrak{M}$ has degree $m$ and an ordinary matric 
basis $e_{11}, \cdots, e_{i j}, \cdots, e_{m m}, \mathfrak{N}$ has degree two and an ordinary matric basis $g_{11}, g_{12}, g_{21}, g_{22}$. Then every quantity of $\mathfrak{S}$ is uniquely expressible in the form

$$
a=A g_{11}+B g_{12}+C g_{21}+D g_{22},
$$

for $A, B, C, D$ in $\mathfrak{M}$, and the transformation of transposition in $\subseteq$ may be so chosen that $a^{\prime}=A^{\prime} g_{11}+C^{\prime} g_{12}+B^{\prime} g_{21}+D^{\prime} g_{22}$. The unity quantity $I$ of $\subseteq$ is the unity quantity $g_{11}+g_{22}$ of both $\mathfrak{M}$ and $\mathfrak{R}$ and every nonsingular skew quantity $t_{1}$ of $\mathfrak{S}$ is congruent to $t=g_{12}-g_{21}$. Define

$$
a^{J}=t a^{\prime} t^{-1}
$$

and see that $\mathfrak{S}_{J}$ consists of all quantities (1) with

$$
D=A^{\prime}, \quad B^{\prime}=-B, \quad C^{\prime}=-C .
$$

Clearly the intersection of $\mathfrak{S}_{J}$ and $\mathfrak{M}$ is the set of $A=A^{\prime}$ in $\mathfrak{M}$.

By Lemma 4, the algebra $\left(\mathfrak{S}_{J}\right)^{*}$ contains $\mathfrak{M}$. If $m=1$ the skew matrices $B$ and $C$ are zero, every $A^{\prime}=A, \mathfrak{S}_{J}=\mathfrak{M}$ has order one, and $\left(\mathfrak{S}_{J}\right)^{*} \neq \mathfrak{S}_{\text {. If }}$ $m>1$ we see that $\left(\Im_{J}\right)^{*}$ contains $e_{j j}\left(e_{j i}-e_{i j}\right) g_{12}=e_{j i} g_{12}$ for $i \neq j$, as well as $e_{i j}\left(e_{j i} g_{12}\right)=e_{i i} g_{12}$ for every $i$. Hence $\left(\Im_{J}\right)^{*}$ contains $g_{12}=\sum_{i} e_{i i} g_{12}$. Similarly $\left(\widetilde{S}_{J}\right)^{*}$ contains $g_{21}, g_{12} g_{21}=g_{11}, g_{21} g_{12}=g_{22},\left(\widetilde{S}_{J}\right)^{*}=\mathfrak{S}$.

If $J$ is an involution over $\mathfrak{F}$ of any simple algebra $\mathfrak{S}$ of degree $n$ over its centrum $\mathfrak{F}$ then there exists a splitting field $\Re$ (of finite degree over $\mathfrak{F}$ ) of $\mathfrak{S}$ such that $\widetilde{S}_{\Omega}$ is a total matric algebra. By Lemma 1 we may regard $J$ as being defined in $\mathfrak{S}_{\boldsymbol{l}}$. Thus we have $a^{J}=t a^{\prime} t^{-1}$ where $t= \pm t^{\prime}$ is in $\mathfrak{S}_{\Re}$ (cf. Albert [2, p. 154]). If $\Omega_{1}$ is any other splitting field of $\subseteq$ and $a^{J}=t_{1} a^{\prime} t_{1}^{-1}$, the quantities $t$ and $t_{1}$ differ by a factor in the composite of $\Omega_{\text {and }} \Omega_{1}$ and $t_{1}$ is symmetric or skew symmetric according as $t$ is symmetric or skew. Let us then call $J$ symmetric or skew in the respective cases.

If $J$ is symmetric there exists a splitting field $\Omega$ of $\subseteq$ such that $t$ is congruent in $\Omega$ to the unity quantity of $\mathscr{S}_{\Omega}, J$ is cogredient in $\Omega$ to transposition. Then $\left(\widetilde{S}_{J}\right)_{\Omega}=\left(\widetilde{S}_{\Omega}\right)_{J}$ is isomorphic to the set of all symmetric matrices over $\Omega$, an algebra of order $n(n+1) / 2 ; \varsigma_{J}$ has order $n(n+1) / 2$. Also by Lemma 4, $\left(\left(\mathfrak{S}_{\Omega}\right)_{J}\right)^{*}=\mathfrak{S}_{\Omega}$, and clearly $\left(\left(\mathfrak{S}_{J}\right)_{\Omega}\right)^{*}=\left(\left(\mathfrak{S}_{\Omega}\right)_{J}\right)^{*}=\mathfrak{S}_{\Omega}$, the order of $\left(\mathfrak{S}_{J}\right)^{*}$ is

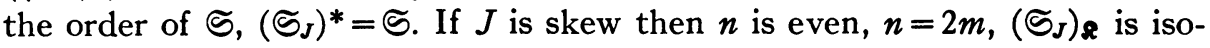
morphic to the set of $n$-rowed matrices defined in (1), (3). The order of this set is $m^{2}+2(m(m-1) / 2)=2 m^{2}-m=n(n-1) / 2$ and, by the argument above, $\left(\Im_{J}\right)^{*}=\Im$ unless $m=1$. We have proved the following theorem.

THEOREM 1. Let $\subseteq$ be a simple associative algebra of degree $n$ over its centrum $\mathfrak{F}$ and $J$ be an involution over $\mathfrak{F}$ of $\mathfrak{S}$. Then the order of the special Jordan algebra $\mathfrak{S}_{J}$ is $n(n+1) / 2$ or $n(n-1) / 2$ according as $J$ is symmetric or skew and $n$ is even in the latter case. Also $\left(\mathfrak{\Im}_{J}\right)^{*}=\mathfrak{\subseteq}$ except when $J$ is skew and $\mathfrak{\Im}_{J}$ has order one.

4. The simplicity of the algebra $\mathfrak{S}^{0}$. The principal result on the special 
Jordan algebra $\mathfrak{S}^{0}$ obtained by quasimultiplication from an associative algebra $\mathfrak{S}$ is:

THEOREM 2. The special Jordan algebra $\mathfrak{S}^{0}$ is simple if and only if $\mathfrak{S}$ is simple.

If $\mathfrak{B}$ is an ideal of $\subseteq$ the products $a b$ and $b a$ are in $\mathscr{B}$ for every $b$ of $\mathscr{B}$ and $a$ of $\mathfrak{S},(a b+b a) / 2$ is also in $\mathfrak{B}, \mathfrak{B}^{0}$ is an ideal of $\mathfrak{S}^{0}$. If $\mathfrak{S}^{0}$ is simple the linear space $\mathfrak{B}^{0}$ is zero or $\mathfrak{S}^{0}, \mathfrak{B}$ is zero or $\mathfrak{S}, \mathfrak{S}$ is simple. Conversely let $\subseteq$ be simple. Then it is clear that not only $\subseteq$ but also $\mathfrak{S}^{0}$ may be regarded as an algebra over the centrum of $\subseteq$ and we may assume that $\subseteq$ is a normal simple algebra over $\mathfrak{F}$. Suppose first that $\subseteq$ is a total matric algebra and that $e_{11}, \cdots$, $e_{i j}, \cdots, e_{n n}$ form an ordinary matric basis of $\mathfrak{S}$. Then if $\mathfrak{B}$ is a nonzero ideal of $\mathfrak{S}^{0}$ and $b=\sum_{i j} \beta_{i j} e_{i j} \neq 0$ is $\mathrm{n} \mathscr{B}$ so is $e_{i i} b+b e_{i i}=\sum_{j} \beta_{i j} e_{i j}+\sum_{k} \beta_{k i} e_{k i}$. Also $e_{j j}\left(e_{i i} b+b e_{i i}\right)+\left(e_{i i} b+b e_{i i}\right) e_{j j}=\beta_{j i} e_{j i}+\beta_{i j} e_{i j}$ is in $\mathscr{B}$ for every $i$ and $j \neq i$. If some $\beta_{i i} \neq 0$ the ideal $\mathscr{B}$ contains $2 \beta_{i i} e_{i i}$ and hence $e_{i i}$. Otherwise some $\beta_{j i} \neq 0$ for $i \neq j, b_{0}=e_{i j}\left(\beta_{j i} e_{j i}+\beta_{i j} e_{i j}\right)+\left(\beta_{j i} e_{j i}+\beta_{i j} e_{i j}\right) e_{i j}=\beta_{j i} e_{i i}+\beta_{j i} e_{j j}$ is in $\mathscr{B}$ and by the process above we have a quantity $e_{i i}$ in $\mathscr{B}$. Then $e_{i i} e_{i j}+e_{i j} e_{i i}=e_{i i}$ if $i \neq j$ and hence $\mathfrak{B}$ contains every $e_{i j}$ for our fixed $i$. Similarly $\mathscr{B}$ contains $e_{j i} e_{i i}+e_{j i} e_{j i}=e_{j i}$ and $e_{i j} e_{j i}+e_{j i} e_{i j}-e_{i i}=e_{j j}$. It follows that $\mathscr{B}$ contains every $e_{j j}$ and, by the process above, every $e_{i j}, \mathfrak{B}=\mathfrak{S}^{0}, \mathfrak{S}^{0}$ is simple. If now $\mathfrak{S}$ is any simple algebra over its centrum $\mathfrak{F}$ there exists a scalar extension $\Re$ of $\mathfrak{F}$ such that $\mathfrak{S}_{\Omega}$ is a total matric algebra and hence $\left(\widetilde{S}_{\Omega}\right)^{0}$ is normal simple. But $\left(\widetilde{S}_{\Omega}\right)^{0}=\left(\widetilde{S}^{0}\right)_{\Omega}$ is then simple and is indeed normal simple - that is, simple for every scalar extension $\Omega$ of $\mathfrak{F}$-and the theorem is proved.

Corollary. The algebra $\mathfrak{S}^{0}$ is normal simple if and only if $\mathfrak{S}$ is normal simple.

5. The algebras $\mathfrak{S}_{J}$. Let $\subseteq$ be a total matric algebra of degree $n$ and $J$ be the transformation of transposition so that $\widetilde{S}_{J}$ has a basis consisting of

$$
f_{i j}=f_{j i}=\left(e_{i j}+e_{j i}\right) / 2 \quad(i, j=1, \cdots, n) .
$$

Multiplication in the commutative algebra $\mathcal{S}_{J}$ is defined by

$$
\begin{aligned}
& f_{i i}^{2}=f_{i i}, \quad 4 f_{i j}^{2}=f_{i i}+f_{i j}, \quad 4 f_{i j} \cdot f_{i k}=f_{j k}, \\
& 2 f_{i i} \cdot f_{i k}=f_{i k}, \quad f_{i i} \cdot f_{i j}=f_{i i} \cdot f_{t k}=f_{i j} \cdot f_{t k}=0
\end{aligned}
$$

for $i, j, k, t$ all distinct and ranging from 1 to $n$. If $\mathfrak{B}$ is an ideal of $\mathfrak{S}_{J}$ and $b=\sum_{i j} \beta_{i j} f_{i j} \neq 0$ is in $\mathscr{B}$, so are $2 f_{i i} \cdot b=\sum_{i \star j} \beta_{i j} f_{i j}+2 \beta_{i j} f_{i i}$ and $\left(2 f_{i i} \cdot b\right) \cdot\left(2 f_{j j}\right)$ $=\beta_{i j} f_{i j}$ for $i \neq j$. If all $\beta_{i j}=0$ then there is some $\beta_{i i} \neq 0, b=\sum_{j} \beta_{j i} f_{j j}$, $f_{i i}$ is in $\mathscr{B}$ for some $i$, and $2 f_{i i} \cdot f_{i j}=f_{i j}$ is in $\mathfrak{B}$. Hence there is some $f_{i j}$ in $\mathfrak{B},\left(2 f_{i j}\right)^{2}=f_{i i}+f_{j j}$ is in $\mathscr{B}$ and $f_{i i}=f_{i i} \cdot\left(f_{i i}+f_{j j}\right)$ is in $\mathscr{B}$. So is $2 f_{i i} \cdot f_{i k}=f_{i k},\left(2 f_{i k}\right)^{2}=f_{i i}+f_{k k}$ and the difference $f_{k k}$ is also in $\mathfrak{B}$. Then $\mathscr{B}$ contains every $f_{i i}, \mathfrak{B}$ contains every $2 f_{i i} \cdot f_{i j}=f_{i j}, \mathfrak{B}=\mathfrak{S}_{J}, \mathfrak{S}_{J}$ is simple.

We next let $J$ be defined as in (2) so that if $m=1$ the algebra $\widetilde{S}_{J}$ is iso- 
morphic to $\mathfrak{F}$ and is simple. Hence let $m>1$ and $a=A g_{11}+A^{\prime} g_{22}+B g_{12}+C g_{21}$. Then if $G$ is in $\mathfrak{M}, H$ and $K$ are skew quantities of $\mathfrak{M}$ and we have the quasiproducts

$$
\begin{aligned}
\left(2 G g_{11}+2 G^{\prime} g_{22}\right) \cdot a= & (G A+A G) g_{11}+(G A+A G)^{\prime} g_{22} \\
& +\left(G B+B G^{\prime}\right) g_{12}+\left(G^{\prime} C+C G\right) g_{21}, \\
\left(2 H g_{12}\right) \cdot a= & H C g_{11}+(H C)^{\prime} g_{22}+\left(H A^{\prime}+A H\right) g_{12}, \\
\left(2 K g_{21}\right) \cdot a= & B K g_{11}+(B K)^{\prime} g_{22}+\left(K A+A^{\prime} K\right) g_{21} .
\end{aligned}
$$

Let $\mathfrak{B}$ be a nonzero ideal of $\mathfrak{S}_{J}$ and $b=A_{0} g_{11}+A_{0}^{\prime} g_{22}+B_{0} g_{12}+C_{0} g_{21} \neq 0$ be in $\mathfrak{B}$. If $A_{0}=0$ then $B_{0}$ and $C_{0}$ are not both zero. If $B_{0} \neq 0$ we can write $B_{0}=P B_{1} P^{\prime}$ where $B_{1}^{2} \neq 0, K=P^{\prime-1} B_{0} P^{-1} \neq 0$ is skew, $B_{0} K=P B_{1}^{2} P^{-1} \neq 0$. Then we use (8) to see that $\left(2 K g_{21}\right) \cdot b$ is in $\mathscr{B}$ and the coefficient of $g_{11} \neq 0$. Similarly if $C_{0} \neq 0$ we may choose $H$ so that $H C_{0} \neq 0$. Hence there is a quantity $b \neq 0$ in $\mathscr{B}$ such that $A_{0} \neq 0$. Now $\mathfrak{B}$ contains all $\left(2 G g_{11}+2 G^{\prime} g_{22}\right) \cdot b$ and the coefficient of $g_{11}$ is $G A_{0}$. The set of all such quantities contains $A_{0}$ and is a nonzero ideal $\mathfrak{B}_{1}$ of $\mathfrak{M}^{0}$. By Theorem $2, \mathfrak{M}^{0}$ is simple, $\mathfrak{B}_{1}=\mathfrak{M}^{0}$, there is a quantity

$$
d=g_{11}+g_{22}+B_{2} g_{12}+C_{2} g_{21}
$$

which is in $\mathfrak{B}$. If $B_{2} C_{2}=I$ (the unit matrix) we form the quasiproduct $\left(2 B_{2} g_{12}\right) \cdot d$ by the use of (7) to get $d_{0}=g_{11}+g_{22}+2 B_{2} g_{12}$, and, by (8), we form $\left(C_{2} g_{21}\right) \cdot d_{0}$ and obtain

$$
d_{1}=g_{11}+g_{22}+C_{2} g_{21} \text {. }
$$

Then since $d_{0}$ and $d_{1}$ are in $\mathscr{B}$ we have $d_{0}-2\left(d-d_{1}\right)=g_{11}+g_{22}$ is also in $\mathscr{B}$ and $\mathfrak{B}=\mathfrak{S}_{J}$ in this case. Hence we may assume $B_{2} C_{2} \neq I$. We form a quantity $\left(B_{2} g_{12}+C_{2} g_{21}\right) \cdot d$ by the use of $(7)$ and $(8)$ to get

$$
h=B_{2} C_{2} g_{11}+\left(B_{2} C_{2}\right)^{\prime} g_{22}+B_{2} g_{12}+C_{2} g_{21}
$$

and we have $d-h=A_{2} g_{11}+A^{\prime} g_{22}$ in $\mathfrak{B}$ where $A_{2}=I-B_{2} C_{2} \neq 0$. Then $\mathfrak{B}$ contains all $\left(G A_{2}+A_{2} G\right) g_{11}+\left(G A_{2}+A_{2} G\right)^{\prime} g_{22}$ and, as in the proof above, every $A g_{11}+A^{\prime} g_{22}$. Using (7) and (8) we see that $B$ contains every $B g_{12}$ and $C g_{21}$, $\mathfrak{B}=\mathfrak{S}_{\text {J }}$.

We may now prove the following theorem.

THEOREM 3. The special Jordan algebras $\mathfrak{S}_{J}$ of Theorem 1 are normal simple algebras.

For there exists a scalar extension $\Omega$ of the centrum $\mathfrak{F}$ of $\mathcal{S}$ such that $\widetilde{S}_{\boldsymbol{R}}$ is a total matric algebra, $J$ in $\widetilde{S}_{\boldsymbol{R}}$ is cogredient to one of the involutions $J_{0}$ above, $\left(\mathfrak{S}_{R}\right)_{J}$ is isomorphic to $\left(\boldsymbol{S}_{R}\right)_{J_{0}}$ and is simple. If $\mathscr{B}$ is an ideal of $\mathfrak{S}_{J}$ the space $\mathfrak{B}_{R}$ is an ideal of $\left(\mathfrak{S}_{J}\right)_{R}=\left(\mathfrak{S}_{R}\right)_{J}$ and is zero or $\left(\mathfrak{S}_{J}\right)_{R}, \mathfrak{B}$ is zero or $\mathfrak{S}_{J}$, $\mathfrak{S}_{J}$ is simple. Since $\widetilde{S}_{\Re}$ is simple for every scalar extension $\Re$ of the centrum $\mathfrak{F}$ of $\mathfrak{S}_{\text {so }}$ is $\left(\mathfrak{S}_{J}\right)_{\Omega}$, and our theorem is proved. 
6. Extension of isomorphisms. If a linear subspace $\mathfrak{A}$ of an associative algebra $\mathfrak{S}$ over $\mathfrak{F}$ is a special Jordan algebra any automorphism $T$. of $\mathfrak{S}$ carries each quantity $a$ of $\mathfrak{A}$ into a quantity $a^{T}$ such that

$$
2(a \cdot b)^{T}=(a b+b a)^{T}=\left(a^{T} b^{T}+b^{T} a^{T}\right)=2 a^{T} \cdot b^{T} .
$$

Hence the linear mapping $a \rightarrow a^{T}$ is an isomorphism of $\mathscr{A}$ and the special Jordan algebra $\mathfrak{A}^{\boldsymbol{T}}$ of all the $a^{\boldsymbol{T}}$. Moreover in some cases we may prove conversely that if $\mathfrak{A}$ and $\mathfrak{B}$ are isomorphic special Jordan algebras which are subspaces of $\mathfrak{S}$ then there is an automorphism $T$ of $\subseteq$ such that $\mathbb{B}=\mathscr{Q}^{T}$ and $a \rightarrow a^{T}$ is the given isomorphism. Evidently our hypothesis is that $T$ is a linear mapping of a prescribed subspace $\mathfrak{A}$ of $\mathfrak{S}$ on a second linear subspace $\mathfrak{A}^{\boldsymbol{T}}$ such that

$$
(a b+b a)^{T}=a^{T} b^{T}+b^{T} a^{T}
$$

for $a$ and $b$ in $\mathfrak{A}$, and we wish to define a linear transformation $U$ on $\mathfrak{S}$ such that $a^{U}=a^{T},(b c)^{U}=b^{U} c^{U}$ for every $a$ of $\mathfrak{A}$ and every $b$ and $c$ of $\mathfrak{S}$. We begin our investigation by proving the following lemma.

Lemma 5. If $e$ is any idempotent of $\mathfrak{A}$ the quantity $e^{T}$ is idempotent. If $e$ and $f$ are orthogonal idempotents so are $e^{T}$ and $f^{T}$.

For, by (9), $\left(e^{2}\right)^{T}=\left(e^{T}\right)^{2}=e^{T}$ if $e^{2}=e$. If $e f=f e=0$ we use (9) to get $e f+f e=0=0^{T}=e^{T} f^{T}+f^{T} e^{T}$. Then $e^{T}\left(e^{T} f^{T}+f^{T} e^{T}\right)=e^{T} f^{T}+e^{T} f^{T} e^{T}=\left(e^{T} f^{T}+f^{T} e^{T}\right) e^{T}$ $=e^{T} f^{T} e^{T}+f^{T} e^{T}, e^{T} f^{T}=f^{T} e^{T}=-f^{T} e^{T}=0$ as desired.

We now consider the algebra $\varsigma_{J}$ of $(4),(5)$ and have

$$
\begin{gathered}
e_{i i}=f_{i i}, \quad 4\left(f_{i j}^{T}\right)^{2}=f_{i i}^{T}+f_{j i}^{T}, \\
2\left(f_{i j}^{T} f_{i k}^{T}+f_{i k}^{T} f_{i j}^{T}\right)=f_{j k}^{T}, \quad\left(f_{i i}^{T} f_{i k}^{T}+f_{i k}^{T} f_{i i}^{T}\right)=f_{i k}^{T}, \\
f_{i i}^{T} f_{j i}^{T}+f_{j i}^{T} f_{i i}^{T}=f_{i j}^{T} f_{t k}^{T}+f_{t k}^{T} f_{i j}^{T}=f_{i i}^{T} f_{t k}^{T}+f_{t k}^{T} f_{i i}^{T}=0
\end{gathered}
$$

for $i, j, k, t=1, \cdots, n$ and $i, j, k, t$ all distinct. Define a linear transformation $U$ on $\subseteq$ by

$$
\boldsymbol{e}_{i j}^{U}=f_{j i}^{T}=e_{i j}^{T}, \quad e_{i j}^{U}=2 f_{i i}^{T} f_{i j}^{T} f_{j i}^{T}
$$

for $i \neq j ; i, j=1, \cdots, n$, so that, by Lemma 5 , we have

$$
\left(e_{i i}^{U}\right)^{2}=e_{i i}^{U} \neq 0, \quad e_{i i}^{U} e_{j i}^{U}=0
$$

for $i \neq j ; i, j=1, \cdots, n$. Then by (11) we have

$$
\stackrel{e_{i i} e_{i j}}{U}=\stackrel{e_{i j} e_{j i}^{U}}{U}=e_{i j}^{U}, \quad \underset{e_{i j} e_{t k}^{U}}{U}=0
$$

for $j \neq t ; i, j, t=1, \cdots, n$. We compute $e_{i j}^{U} e_{j k}^{U}=4 e_{t i}^{T} f_{i j}^{T} e_{j j}^{T} f_{j k}^{T} e_{k k}^{T}$ for $j \neq i, k$. Use (10) to obtain

$$
e_{j i j}^{T} f_{j k}^{T}=f_{j k}^{T}-f_{j k}^{T} e_{j i}^{T},
$$


and have $e_{i j}^{U} e_{j k}^{U}=4 e_{i j}^{T} f_{i j}^{T} f_{j k}^{T} e_{k k}^{T}$. If $k \neq i$ we have $2 f_{i j}^{T} f_{j k}^{T}=f_{i k}^{T}-2 f_{j k}^{T} f_{i j}^{T}$ and since $e_{i t}^{T} f_{j k}^{T}+f_{j k}^{T} e_{i i}^{T}=e_{k r}^{T} f_{i j}^{T}+f_{i j}^{T} e_{k i}^{T}=0$ for $i \neq j, i \neq k, k \neq j$ we obtain $e_{i i}^{T} f_{j k}^{T} f_{i j}^{T} e_{k k}^{T}$ $=f_{j k}^{T} e_{i i}^{T} e_{k k}^{T} f_{i j}^{T}=0$,

$$
e_{i j}^{U} e_{j k}^{U}=2 \underset{e_{i i}}{T} f_{i k}^{T} e_{k k}^{T}=e_{i k}^{U}
$$

If $k=i$ we have $4 f_{i j}^{T} f_{i i}^{T}=\left(f_{i i}^{T}+f_{j j}^{T}\right), e_{i j}^{U} e_{j i}^{U}=e_{i i}^{T}\left(e_{i i}^{T}+e_{j j}^{T}\right) e_{i i}^{T}=e_{i t}^{T}$. Hence in all cases we have (12), (13), and $U$ is an automorphism of $\mathfrak{S}$. But $2 f_{i j}^{U}=e_{i j}^{U}+e_{j i}^{U}$ $=2\left(f_{i f}^{T} T_{i j}^{T} f_{j j}^{T}+f_{j j}^{T} f_{i j}^{T} f_{i t}^{T}\right)=f_{i i}^{T}\left(2 f_{i j}^{T} f_{j j}^{T}\right)+\left(2 f_{j j}^{T} f_{i j}^{T}\right) f_{i i}^{T}=2 f_{i t}^{T} f_{i j}^{T}+2 f_{i j}^{T} f_{i i}^{T}=2 f_{i j}^{T}, f_{i j}^{U}=f_{i j}^{T}$ and we have proved that $a \rightarrow a^{U}$ is the given isomorphism of $\mathfrak{A}$ and $\mathfrak{B}$.

Consider next the case where $J$ is defined as in (1), (3). Then as we noted in the proof of Theorem 1 the intersection of $\mathfrak{M}$ and $\mathfrak{S}_{J}$ is the set of all $A g_{11}+A g_{22}=A=A^{\prime}$ in $\mathfrak{M}$. By the proof above there is an isomorphism $U$ of $\mathfrak{M}$ with $\left(\left(\mathfrak{M} \cap \mathfrak{S}_{J}\right)^{T}\right)^{*}$ such that $A^{T}=A^{U}$ for $A=A^{\prime}$ in $\mathfrak{M}$. We may extend $U$ to an automorphism of $\mathbb{S}$ and may replace $\left(\mathfrak{S}_{J}\right)^{T}$ by the isomorphic special Jordan algebra $\left(\mathfrak{S}_{J}\right)^{T U^{-1}}$ and thus replace each $A^{T}$ defined for $A=A^{\prime}$ in $\mathfrak{M}$ by $A$. Hence there is no loss of generality if we assume that $A=A^{T}$ for every symmetric $A$ in $\widetilde{S}_{J}$. We are also studying only the case $m>1$.

The quantities

$$
\begin{array}{rrr}
h_{i j}=e_{i j} g_{11}+e_{j i} g_{22} & (i, j=1, \cdots, m), \\
f_{i j}=\left(e_{i j}-e_{j i}\right) g_{12}=-f_{j i} & (i \neq j ; i, j=1, \cdots, m), \\
d_{i j}=\left(e_{i j}-e_{j i}\right) g_{21}=-d_{j i} & (i \neq j ; i, j=1, \cdots, m)
\end{array}
$$

are in $\mathfrak{S}_{J}$ and, by hypothesis, we have $\left(h_{i j}+h_{j i}\right)^{r}=h_{i j}+h_{j i}$ for $i, j=1, \cdots$, $m$. Since by hypothesis $h_{\mathfrak{t}}^{T}=h_{\mathfrak{t}}$ and since $T$ is an isomorphism between the special Jordan algebras $\varsigma_{J}$ and $\Im_{J}^{T}$ we have $\left(h_{i i} \cdot\left(h_{j j} \cdot a\right)\right)^{T}=h_{i i} \cdot\left(h_{j j} \cdot a^{T}\right)$. Apply this with $a=f_{i j}, d_{i j}, h_{i j}$ in turn and obtain

$$
\begin{aligned}
& 4 h_{i i} \cdot\left(h_{i j} \cdot f_{i j}^{T}=f_{i j}^{T},\right. \\
& 4 h_{i i} \cdot\left(h_{i j} \cdot d_{i j}^{T}\right)=d_{i j,}^{T}, \\
& 4 h_{i i} \cdot\left(h_{i j} \cdot h_{i j}^{T}=h_{i j}^{T}\right.
\end{aligned}
$$

for $i \neq j$. This yields, by direct computation, assuming $T$ to be an automorphism of $\mathfrak{S}_{J}$,

$$
\begin{aligned}
f_{i j}^{T} & =\alpha_{i j} f_{i j}+\beta_{i j} d_{i j}+\gamma_{i j} h_{i j}+\delta_{j i} h_{j i}, \\
d_{i j}^{T} & =\alpha_{i j}^{\prime} f_{i j}+\beta_{i j}^{\prime} d_{i j}+\gamma_{i j}^{\prime} h_{i j}+\delta_{j i}^{\prime} h_{j i}, \\
h_{i j} & =\alpha_{i j}^{\prime \prime} f_{i j}+\beta_{i j}^{\prime \prime} d_{i j}+\gamma_{i j}^{\prime \prime} h_{i j}+\delta_{j i}^{\prime \prime} h_{j i}
\end{aligned}
$$

for $i, j=1, \cdots, m$. But, by $(6),\left(f_{1 i} \cdot\left(h_{1 i}+h_{i 1}\right)\right)^{T}=0=f_{1 i}^{T} \cdot\left(h_{1 i}+h_{i 1}\right),\left(d_{1 i} \cdot\left(h_{i 1}\right.\right.$ $\left.\left.+h_{1 i}\right)\right)^{T}=0=d_{1 i}^{T} \cdot\left(h_{1 i}+h_{i 1}\right)$ and we conclude that

$$
f_{1 i}^{T}=\alpha_{1 i} f_{1 i}+\beta_{1 i} d_{1 i}+\gamma_{1 i} h_{1 i}-\gamma_{1 i} h_{i 1},
$$




$$
d_{1 i}^{T}=\alpha_{1 i}^{\prime} f_{1 i}+\beta_{1 i}^{\prime} d_{1 i}+\gamma_{1 i}^{\prime} h_{1 i}-\gamma_{1 i}^{\prime} h_{i 1}
$$

for $i=2, \cdots, m$. Since, for $k \neq 1, i$, we have $2\left(f_{1 i} \cdot\left(h_{k i}+h_{i k}\right)\right)^{T}=2 f_{11}^{T} \cdot\left(h_{k i}+h_{i k}\right)$ $=f_{1 k}^{T}=\alpha_{1 k} f_{1 k}+\beta_{1 k} d_{1 k}+\gamma_{1 k} h_{1 k}-\gamma_{1 k} h_{k 1}=\alpha_{1 i} f_{1 k}+\beta_{1 i} d_{1 k}+\gamma_{1 i} h_{1 k}-\gamma_{1 i} h_{k 1}$, and similar results for $d_{1 i}$, we can write

$$
\begin{aligned}
& f_{1 i}^{T}=\alpha f_{1 i}+\beta d_{1 i}+\gamma h_{1 i}-\gamma h_{i 1}, \\
& d_{1 i}^{T}=\alpha^{\prime} f_{1 i}+\beta^{\prime} d_{1 i}+\gamma^{\prime} h_{1 i}-\gamma^{\prime} h_{i 1}
\end{aligned}
$$

for $i=2, \cdots, m$. Observe that, by (7) and (8), $f_{11}^{2}=d_{11}^{2}=\left(f_{11}^{T}\right)^{2}=\left(d_{11}^{T}\right)^{2}=0$ and hence

$$
\alpha \beta+\gamma^{2}=\alpha^{\prime} \beta^{\prime}+\gamma^{\prime 2}=0 .
$$

If now $\alpha=0$ then $\gamma=0, \beta \neq 0, f_{1 i}^{T}=\beta d_{1 i}$. Let $V$ be the automorphism of $\mathfrak{S}$

$$
a \rightarrow a^{v}=v a v^{-1}
$$

with $v=g_{11}+g_{12}+\beta g_{21}$, which by direct computation leaves $h_{i j}+h_{j i}$ invariant and which has the further property that

$$
\left(f_{1 i}^{T}\right)^{V}=f_{1 i}
$$

for $i=2, \cdots, m$. If $\alpha \neq 0$ we take $v$ in (17) to be $v=g_{11}+\gamma g_{21}+\alpha g_{22}$, and we see that $V$ leaves $h_{i j}+h_{j i}$ invariant, and again (18) is valid. Thus we may assume

$$
\begin{array}{rlrl}
\left(h_{i j}+h_{j i}\right)^{T} & =h_{i j}+h_{j i} & (i, j & =1, \cdots, m), \\
f_{1 i}^{T} & =f_{1 i} & (i=2, \cdots, m) .
\end{array}
$$

We observe that $2\left(f_{1 i} \cdot d_{1 i}\right)^{T}=2 f_{1 i}^{T} \cdot d_{11}^{T}=2 f_{1 i} \cdot d_{1 i}^{T}=-\left(h_{11}+h_{i i}\right)^{T}=-h_{11}-h_{i i}$ implies by (7) and (15), (16) that $\beta^{\prime}=1, \alpha^{\prime}+\gamma^{\prime 2}=0$. Let now $W$ be an inner automorphism of $\mathfrak{S}$ generated by $w=g_{11}+g_{22}-\gamma^{\prime} g_{12}$. Using direct computation we see that $W$ leaves $\left(h_{i j}+h_{j i}\right)$ and $f_{1 i}$ invariant, and

$$
\left(d_{1 i}^{T}\right)^{W}=d_{1 i} \quad(i=2, \cdots, m) .
$$

This result permits us to assume

$$
\begin{aligned}
& \left(h_{i j}+h_{j i}\right)^{T}=h_{i j}+h_{j i} \\
& (i, j=1, \cdots, m) \text {, } \\
& f_{1 i}^{T}=f_{1 i} \\
& (i=2, \cdots, m) \text {, } \\
& d_{1 i}^{T}=d_{1 i} \\
& (i=2, \cdots, m) \text {. }
\end{aligned}
$$

By (14) we may write

$$
\begin{aligned}
& h_{1 i}^{T}=\alpha_{i} f_{1 i}+\beta_{i} d_{1 i}+\gamma_{i} h_{1 i}+\delta_{i} h_{i 1}, \\
& h_{i 1}^{T}=\alpha_{i}^{\prime} f_{1 i}+\beta_{i}^{\prime} d_{1 i}+\gamma_{i}^{\prime} h_{1 i}+\delta_{i} h_{i 1}
\end{aligned}
$$

for $i=2, \cdots, m$, where, by (19), 


$$
\alpha_{i}^{\prime}=-\alpha_{i}, \quad \beta_{i}^{\prime}=-\beta_{i}, \quad \gamma_{i}+\gamma_{i}=\delta_{i}+\delta_{i}=1 .
$$

By (6) and (19) we compute $2\left(h_{1 i} \cdot\left(h_{1 i}+h_{i 1}\right)\right)^{T}=2 h_{1 i}^{T} \cdot\left(h_{1 i}+h_{i 1}\right)=\left(h_{11}+h_{i i}\right)^{T}$ $=h_{11}+h_{i i}$ and find

$$
\gamma_{i}+\delta_{i}=1
$$

and, similarly,

$$
\gamma_{i}^{\prime}+\delta_{i}^{\prime}=1 .
$$

Using (6) and (19) we find $2\left(h_{1 i} \cdot\left(h_{k i}+h_{i k}\right)\right)^{T}=2 h_{1 i}^{T} \cdot\left(h_{k s}^{T}+h_{i k}\right)=h_{1 k}^{T}=\alpha_{k} f_{1 k}$ $+\beta_{k} d_{1 k}+\gamma_{k} h_{1 k}+\delta_{k} h_{k 1}$ for $k \neq 1, i$ with similar results for $h_{k 1}$. Hence we may write

$$
\begin{aligned}
h_{1 i}^{T} & =\alpha f_{1 i}+\beta d_{1 i}+\gamma h_{1 i}+\delta h_{i 1} \\
h_{i 1}^{T} & =\alpha^{\prime} f_{1 i}+\beta^{\prime} d_{1 i}+\gamma^{\prime} h_{1 i}+\delta^{\prime} h_{i 1} \\
\alpha^{\prime} & =-\alpha, \quad \beta^{\prime}=-\beta, \\
\gamma+\gamma^{\prime} & =\delta+\delta^{\prime}=\gamma+\delta=\gamma^{\prime}+\delta^{\prime}=1 .
\end{aligned}
$$

Since, by (6), $h_{1 \mathrm{i}}^{2}=\left(h_{1 \mathrm{i}}^{\mathrm{T}}\right)^{2}=h_{i 1}^{2}=\left(h_{i 1}^{T}\right)^{2}=0$ we have

$$
\alpha \beta-\gamma \delta=\alpha^{\prime} \beta^{\prime}-\gamma^{\prime} \delta^{\prime}=0, \quad \gamma \delta=\gamma^{\prime} \delta^{\prime} .
$$

If we compute $\left(h_{1 i} \cdot f_{1 i}\right)^{T}=\left(h_{1 i} \cdot d_{1 i}\right)^{T}=0=h_{1 i}^{T} \cdot f_{1 i}=h_{1 i}^{T} \cdot d_{1 i}$ and use (7), (8), (20), (21) we find

$$
\alpha=\beta=\alpha^{\prime}=\beta^{\prime}=0, \quad \gamma \delta=\gamma^{\prime} \delta^{\prime}=\gamma(1-\gamma)=\gamma^{\prime}\left(1-\gamma^{\prime}\right)=0
$$

and therefore

$$
\gamma=0, \quad \gamma^{\prime}=1
$$

or

$$
\gamma=1, \quad \gamma^{\prime}=0
$$

for $\gamma=0, \gamma^{\prime}=0$ or $\gamma=\gamma^{\prime}=1$ would contradict the last line of (20). If $h_{18}^{T}=h_{i 1}$, $h_{i 1}^{T}=h_{1 i}$ we would have $h_{i k}^{T}=2\left(h_{i 1} \cdot h_{1 k}\right)^{T}=2 h_{1 i} \cdot h_{k 1}=h_{k i}, 2\left(f_{1 i} \cdot h_{i k}\right)^{T}=0=2 f_{1 i} \cdot h_{k i}$ $=f_{1 k}$ which is impossible for $k \neq 1, i$. Therefore

$$
h_{1 i}^{T}=h_{1 i}, \quad h_{i 1}^{T}=h_{i 1} .
$$

Using

$$
\begin{array}{rrr}
h_{i j}^{T}=-2 f_{1 i}^{T} \cdot d_{i j}^{T} & (i, j=1, \cdots, m), \\
f_{i j}^{T}=-2 f_{1 i}^{T} \cdot h_{j 1}^{T} & (i \neq j ; i, j=1, \cdots, m), \\
d_{i j}^{T}=-2 d_{1 i}^{T} \cdot h_{1 j}^{T} & (i \neq j ; i, j=1, \cdots, m),
\end{array}
$$

we see now that $h_{i j}^{T}=h_{i j}, f_{i j}^{T}=f_{i j}, d_{i j}^{T}=d_{i j}$, and this proves that there exists 
an inner automorphism $R$ of $\mathfrak{S}$ inducing the originally given automorphism $T$ of $\mathfrak{S}_{J}$, namely $R=W V U$.

We may now prove the following theorem.

THEOREM 4. Let $\subseteq$ be a simple associative algebra over its centrum $\mathfrak{F}, J$ and $K$ two involutions of $\mathfrak{S}$ over $\mathfrak{F}$. Then each isomorphism $T$ over $\mathfrak{F}$ between $\mathfrak{S}_{J}$ and $\Im_{K}$ of order greater than 1 can be extended uniquely to an automorphism $U$ over $\mathfrak{F}$ of $\mathfrak{S}$. If $J=K$ then the automorphism $U$ which is the extension of the given automorphism of $\mathfrak{S}_{J}$ is generated by a.J-orthogonal element $u$ of $\mathfrak{S}$, that is, $u u^{J}=\alpha$ in $\mathfrak{F}$.

For there exists a scalar extension $\mathfrak{R}$ of the centrum $\mathfrak{F}$ of $\mathfrak{S}$ such that $\mathfrak{S}_{\mathbb{R}}$ is a total matric algebra, $J$ and $K$ can be extended to $\Im_{\&}$ by Lemma 1 , and the extensions $\left(\mathfrak{S}_{J}\right)_{\mathbb{R}}$ and $\left(\mathfrak{S}_{K}\right)_{\mathbb{R}}$ will be isomorphic. In $\mathfrak{S}_{\mathfrak{R}}$ both $J$ and $K$ are cogredient to one of the involutions $J_{0}$ above, for otherwise, by Theorem 1, $\mathfrak{S}_{J}$ and $\mathfrak{\Im}_{K}$ would not have the same order as linear spaces over $\mathfrak{F}$. The isomorphism between $\left(\mathfrak{S}_{\mathbb{R}}\right)_{J}$ and $\left(\mathfrak{S}_{\mathbb{R}}\right)_{K}$ induces an automorphism of $\left(\mathfrak{S}_{\mathbb{R}}\right)_{J_{0}}$ (Lemma 3) which by the above argument can be extended to an automorphism of $\left(\mathfrak{S}_{J}\right)^{*}=\left(\mathfrak{S}_{K}\right)^{*}=\mathfrak{S}_{\text {. }}$.

The uniqueness follows from Theorem 1 . For if $a_{1}, \cdots, a_{s}$ is a basis of $\mathfrak{S}_{J}$ over $\mathfrak{F}$ then every element $a$ of $\mathfrak{S}$ has the form $a=\sum \alpha a_{i_{1}} \cdots a_{i_{k}}$ for $\alpha$ in $\mathfrak{F}$ and if $U$ and $V$ are two extensions of $T$ then $a^{U}=\sum \alpha a_{i_{1}}^{T} \cdots a_{i_{k}}^{T}=a^{V}, U=V$.

If $J=K$ we have, for each $a$ of $\mathfrak{S}$, by Theorem $1, a^{J U}=\sum \alpha a_{i_{k}}^{T} \cdots a_{i_{1}}^{T}$ $=a^{U J}, a^{J U}=u a^{J} u^{-1}=a^{U J}=\left(u^{-1}\right)^{J} a^{J} u^{J}$ and hence $u u^{J}=\alpha_{0}$ in $\mathfrak{F}$. This completes the proof of the theorem.

Consider now two simple associative algebras $\Re$ and $\subseteq$ over their common centrum $\mathfrak{F}$, and $J$ and $K$ involutions of $\Re$ and $\mathfrak{S}$, respectively. Assume also that $\Re_{J}$ and $\mathfrak{S}_{K}$ are isomorphic. There exists a scalar extension field $\mathfrak{R}$ of $\mathfrak{F}$ such that $J$ in $\Re_{\mathbb{R}}$ is cogredient to one of the involutions $J_{0}$ above, and at the same time $K$ in $\mathfrak{S}_{\&}$ is cogredient to the same $J_{0}$ for otherwise $\Re_{J}$ and $\mathfrak{S}_{K}$ could not be isomorphic. Thus by Lemma $3,\left(\Re_{R}\right)_{J}$ is isomorphic to $\left(\Re_{\varepsilon}\right)_{J_{0}}$ and so is $\left(S_{R}\right)_{K}$. The isomorphism between $\left(\Re_{R}\right)_{J}$ and $\left(\mathscr{S}_{R}\right)_{K}$ thus induces an automorphism of $\left(\Re_{q}\right)_{J_{0}}$ which by Theorem 4 can be extended to an automorphism $U$ of $\Re_{\varepsilon}$ which will induce the desired isomorphism between $\left(\Re_{J}\right)^{*}$ and

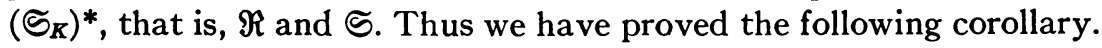

CoROLlaRY. Let $\Re$ and $\subseteq$ be two simple associative algebras over the same centrum $\mathfrak{F}, J$ and $K$ involutions over $\mathfrak{F}$ of $\Re$ and $\mathfrak{S}$, respectively. Then each isomorphism $T$ between $\Re_{J}$ and $\mathfrak{S}_{K}$ of order greater than one can be extended to an isomorphism $U$ between $\left(\Re_{J}\right)^{*}=\Re$ and $\left(\mathfrak{S}_{K}\right)^{*}=\mathfrak{S}$.

Lemma 3 and Theorem 4 imply the following theorem.

THEOREM 5. Let $\mathfrak{S}$ be a simple associative algebra over its centrum $\mathfrak{F}$. Then given two involutions $J$ and $K$ of $\mathfrak{S}$ over $\mathfrak{F}, \mathfrak{S}_{J}$ and $\mathfrak{\Im}_{K}$ are isomorphic if and only if $J$ and $K$ are cogredient, provided $\mathfrak{S}_{J}$ and $\mathfrak{\Im}_{K}$ have order greater than one. 
If $J$ and $K$ are cogredient then $\mathfrak{\subseteq}_{J}$ and $\mathfrak{\subseteq}_{K}$ are isomorphic by Lemma 3. Suppose that $\mathfrak{S}_{J}$ and $\mathfrak{\Im}_{K}$ are isomorphic. Then the isomorphism $T$ between $\mathfrak{S}_{J}$ and $\mathfrak{S}_{K}$ can be extended, by Theorem 4 , to an automorphism of $\varsigma$ wnich we shall call $U$. By Theorem 1, every $a$ of $\subseteq$ is of the form $a=\sum \alpha a_{i_{1}} \cdots a_{i_{k}}$ for $\alpha$ in $\mathfrak{F}$ and the $a_{i}$ are a basis of $\mathfrak{S}_{J}$ over $\mathfrak{F}$. Then $a^{J U}=\sum \alpha a_{i_{k}}^{T} \cdots a_{i_{1}}^{T}=a^{U K}$, $J U=U K, J$ and $K$ are cogredient.

Two $J$-orthogonal elements $g$ and $f$ of an associative simple algebra $\mathfrak{S}$ generate the same automorphism of $\mathfrak{S}_{J}$ if and only if $g=\alpha f$ for some $\alpha$ in $\mathfrak{F}$, for $\mathrm{gag}^{-1}=f a f^{-1}$ for all $a$ in $\mathfrak{S}_{J}$ implies the same equation for all $a$ in $\subseteq$ by Theorem 1, provided that the order of $\widetilde{S}_{J}$ be greater than one; but $g a g^{-1}=f a f^{-1}$ for all $a$ of $\subseteq$ implies that $g=\alpha f$ for some $\alpha$ in $\mathfrak{F}$. We have proved the following theorem.

THEOREM 6. Let $\mathfrak{S}$ be a simple associative algebra over its centrum $\mathfrak{F}, J$ be an involution over $\mathfrak{F}$ of $\mathfrak{S}$ such that the order of $\mathfrak{S}_{J}$ is greater than one. Then the group of automorphisms of $\mathfrak{S}_{J}$ over $\mathfrak{F}$ is isomorphic to the group $\mathfrak{S}^{*} / \mathfrak{F}^{*}$ where $\mathfrak{S}^{*}$ is the multiplicative group of all regular J-orthogonal elements of $\mathfrak{S}$ and $\mathfrak{F}^{*}$ is the multiplicative group of $\mathfrak{F}$.

7. Classification of the algebras $\mathfrak{S}_{J}$ over special fields. Theorem 5 and the fact that cogredience of $J_{0} T$ and $J_{0} U$, for $J_{0}$ an involution and $T, U$ automorphisms of $\mathfrak{S}$, is equivalent to $J_{0}$-congruence of the generating elements $t$ and $\alpha u$ for $\alpha$ in $\mathfrak{F}$ (cf. Albert [2, p. 154]) imply that if $\mathfrak{S}$ is a simple associative algebra over its centrum $\mathfrak{F}$, the classification of its special Jordan algebras $\widetilde{S}_{J}$ is equivalent to the classification of $J_{0}$-symmetric and $J_{0}$-skew elements of $S$ as to $J_{0}$-congruence. This depends on the structure of the ground field $\mathfrak{F}$. If $\mathfrak{F}$ is an algebraically closed field then every $J$ is cogredient either to the operation of transposition of matrices (for we may consider $\subseteq$ as a total matric algebra over $\mathfrak{F}$ ) or $J$ is cogredient to the involution defined by (1) and (3) (cf. Albert [1, pp. 108, 110]).

If $\mathfrak{F}$ is a real closed field it is necessary to distinguish the case where $\mathfrak{S}$ is a total matric algebra over $\mathfrak{F}$ from that where $\mathfrak{S}$ is a total matric algebra with quaternionic elements. In the first case if $J$ denotes matrix transposition the $J$-symmetric elements are $J$-congruent to

$$
\left(\begin{array}{cc}
I_{p} & 0 \\
0 & -I_{n-p}
\end{array}\right)
$$

where we take $p \geqq n / 2$ since we are permitted to multiply with real factors (cf. Albert $[1$, p. 114]). There are $[n / 2]+1$ types of nonisomorphic special Jordan algebras in this case. In the case of $J$-skew elements there is only c ne possibility, namely, the degree of the algebra $\subseteq$ is an even number $n=2 m$ and $J$ is given by (1) and (3) (cf. Albert [1, p. 108]).

In case we are dealing with real quaternionic matrices it is best to take for $J$ the correspondence $a \rightarrow \bar{a}^{\prime}$ where $a^{\prime}$ is the transpose of $a$ and $\bar{a}$ is the ma- 
trix of the conjugate elements of $a$. In the symmetric case we again obtain (22) (cf. Jacobson [5, p. 546]) and there are $[n / 2]+1$ types of nonisomorphic special Jordan algebras. In the skew case we obtain only one type given by

$$
\left(\begin{array}{llll}
t & & & \\
& t & & \\
& & \cdot & \\
& & & t
\end{array}\right)
$$

where $t$ is a quaternion such that $\bar{t}=-t$ (cf. Jacobson, loc. cit.).

8. The main theorem. While it is easy to see what happens to an algebra upon extension of its base field it is quite difficult to determine the structure of an algebra from that of one of its scalar extensions. Thus, if $\mathfrak{A}$ over $\mathfrak{F}$ is a special Jordan algebra $\mathfrak{S}_{J}$ for some associative algebra $\mathfrak{S}$ over $\mathfrak{F}$ with an involution $J$, it is clear, by Lemma 1 , that for every extension field $\mathfrak{R}$ of $\mathfrak{F}$, $\mathfrak{A}_{R}=\left(\mathfrak{S}_{J}\right)_{\mathbb{R}}=\left(\mathfrak{S}_{8}\right)_{J}$ is again a special Jordan algebra of the same kind. We shall show that in certain cases we can prove a converse theorem, namely that if, given an algebra $\mathfrak{A}$ over $\mathfrak{F}$, there exists a scalar extension field $\mathfrak{R}$ of $\mathfrak{F}$ such that $\mathscr{A}_{\mathbb{R}}$ is isomorphic to a special Jordan algebra $\mathfrak{S}_{J}$ for $\mathfrak{S}_{\text {over }} \mathfrak{R}$, then there exists an associative algebra $\mathfrak{I}$ over $\mathfrak{F}$ with an involution $J^{\prime}$ such that $\mathfrak{A}$ is isomorphic to $\mathfrak{I}^{\prime \prime}$.

Consider an algebra $\mathfrak{A}$ over $\mathfrak{F}$ and assume that there exists a normal separable scalar extension field $\mathfrak{R}$ of $\mathfrak{F}$ such that $\mathfrak{A}_{\mathbb{Z}}$ is isomorphic to $\mathfrak{S}_{J}$ where $\mathfrak{S}$ is a total matric algebra over $\mathbb{R}$, and $J$ is an involution of $\mathfrak{S}$ over $\mathbb{R}$ cogredient either to transposition $J_{1}$ or to $J_{2}$ as defined by (1) and (3). If $s$ is in $\mathfrak{S}$, we can write $s$ as the matrix $\left(s_{i j}\right)$ with $s_{i j}$ in $\mathfrak{R}$ and if $t$ is in the Galois group $\mathbb{B}$ of $\mathfrak{R}$ over $\mathfrak{F}$, we can introduce the following linear map of $\subseteq$ unto itself: $s=\left(s_{i j}\right) \rightarrow s^{t}=\left(s_{i j}^{i}\right)$ where $s_{i j}^{t}$ is the map of $s_{i j}$ under $t$ of $\mathbb{G}$. This map is an automorphism of $\mathfrak{S}$ over $\mathfrak{F}$. It is clear that $t$ commutes with $J_{1}$. If $s$ is written as in (1) we have

$$
s^{t}=A^{t} g_{11}+B^{t} g_{12}+C^{t} g_{21}+D^{t} g_{22}
$$

where the matrices $A^{t}, B^{t}, C^{t}, D^{t}$ have the obvious meaning as defined above. Again it is clear that $t$ and $J_{2}$ commute. Suppose that $a_{1}, \cdots, a_{m}$ is a basis of $\mathfrak{A}$ over $\mathfrak{F}$, and hence of $\mathfrak{A}_{\mathbb{q}}$ over $\mathfrak{l}$. Denote by $b_{1}, \cdots, b_{m}$ the corresponding matrices of $\mathfrak{S}$ forming a basis of $\mathfrak{S}_{J}$ over $\mathbb{R}$. The elements $a$ in $\mathfrak{S}_{J}$ have the form $a=\sum c_{i} b_{i}$ with $c_{i}$ in $\&$. Then $a \rightarrow a^{T}=\sum c_{i} b_{i}^{i}$ defines an automorphism of $\mathfrak{S}_{J}$ over $\mathfrak{R}$ which, by Theorem 4 , can be extended to an automorphism of $\mathfrak{S}$ over $\&$ which we shall also denote by $T$. Again we have $T J_{1}=J_{1} T, T J_{2}=J_{2} T$.

Consider the set $\mathbb{B}$ consisting of all $s$ in $\mathfrak{S}$ such that $s^{t}=s^{T}$ for all $t$ in $(\mathcal{B}$. $\mathscr{B}$ is an associative algebra over $\mathfrak{F}$ as it contains with any $a, b$ also the elements $a b$ and $\alpha a+\beta b$ for $\alpha$ and $\beta$ in $\mathfrak{F}$, and if $\gamma$ is in $\mathcal{R}$ and not in $\mathfrak{F}$, and $a$ is in $\mathfrak{B}$, then $\gamma a$ is not in $\mathscr{B}$, for $(\gamma a)^{t}=(\gamma a)^{T}=\gamma^{t} a^{t}=\gamma a^{T}=\gamma a^{t}$ and $\gamma=\gamma^{t}$ for all $t$ in $\mathbb{S}$, $\gamma$ is in $\mathfrak{F}$. Furthermore, the mappings $J_{1}$ or $J_{2}$ of $\subseteq$ induce mappings $K_{1}$ and 
$K_{2}$ of $\mathscr{B}$, because $t$ and $T$ commute with $J_{1}$ and $J_{2}$ as we have seen above. It is also clear that $J_{i}$ is an extension of $K_{i}$. Moreover $\mathfrak{B}$ contains the $b_{i}$ and hence $\mathfrak{B}_{\boldsymbol{q}}$ contains all expressions $\sum \alpha b_{i_{1}} \cdots b_{i_{k}}, \alpha$ in $\mathfrak{R}$, which, by Theorem 1, constitute S (we may, of course, assume that the order of $\widetilde{S}_{J}$ is greater than one). Thus $\mathfrak{B}_{\mathbb{R}} \supseteq \mathfrak{S}$, and since obviously $\mathfrak{S} \supseteq \mathfrak{B}_{\mathbb{R}}$, we have $\mathfrak{S}=\mathfrak{B}_{\mathbb{8}}$, and $\mathfrak{B}$ is simple over its centrum $\mathfrak{F}$. Let $\mathfrak{A}$ be the special Jordan algebra of matrices whose basis is $b_{1}, \cdots, b_{m}$ over $\mathfrak{F}, \mathfrak{M}$ is isomorphic to $\mathfrak{A}$. Since the $b_{i}$ are in $\mathfrak{B}_{K}$ (for $K=K_{1}$ or $K_{2}$ ) we have $\mathfrak{B}_{K} \supseteq \mathfrak{M}$. Consider now $c$ in $\mathfrak{B}_{K}$. First we have $c^{K}=c$ which means that $c$ is in $\mathfrak{S}_{J}$ and thus $c=\sum c_{i} b_{i}$ for $c_{i}$ in $\mathfrak{R}$. But we also have $c^{t}=c^{T}$ and hence $\sum c_{t} b_{t}^{t}=\sum c_{t}^{t} b_{t}^{t}$. Now the $b_{t}^{t}$ are linearly independent because of the linear independence of the $b_{i}$, and we therefore have $c_{i}=c_{i}^{\imath}$ for all $t$ in $\mathbb{B}, c_{i}$ is in $\mathfrak{F}, c$ is in $\mathfrak{M}, \mathfrak{B}_{K}=\mathfrak{M}$ and $\mathfrak{A}$ is isomorphic to $\mathfrak{B}_{K}$.

We are now in a position to prove the main theorem.

THEOREM 7: Let $\mathfrak{A}$ be an algebra over a nonmodular field $\mathfrak{F}$, and let $\mathfrak{R}$ be a finite scalar extension field of $\mathfrak{F}$. Then if $\mathfrak{A}_{\mathbb{Z}}$ is isomorphic to a special Jordan algebra $\mathfrak{S}_{J}$ for $\mathfrak{S}$ simple over its centrum $\mathbb{\&}$ and having an involution $J$ over $\mathbb{R}$, there exists an associative simple algebra $\mathfrak{B}$ over its centrum $\mathfrak{F}$ with an involution $K$ such that $\mathfrak{B}_{\mathbb{R}}=\mathfrak{S}, J$ is the extension of $K$, and $\mathfrak{A}$ is isomorphic to $\mathfrak{B}_{K}$.

For there exists a finite scalar extension field $\mathfrak{R}^{\prime}$ over $\mathfrak{l}$ such that $\mathfrak{R}^{\prime}$ is normal separable over $\mathfrak{F}, \subseteq_{\mathbb{R}^{\prime}}$ is a total matric algebra, and the extension of $J$ to $S_{\ell^{\prime}}$ is cogredient in $\mathfrak{R}^{\prime}$ either to transposition or to $J$ as defined by (1) and (3). Then $\mathscr{P}_{\mathcal{R}^{\prime}}$ is isomorphic to $\left(\widetilde{S}_{\mathbb{R}^{\prime}}\right)_{J}$ and by the above argument there exists an associative simple algebra $\mathfrak{B}$ over $\mathfrak{F}$ with an involution $K$ such that $\mathfrak{A}$ is isomorphic to $\mathfrak{B}_{K}, \mathfrak{B}_{\mathbb{Z}^{\prime}}=\mathfrak{S}_{\mathbb{R}^{\prime}}, J$ is an extension of $K$. It is clear that this algebra $\mathfrak{B}$ has the properties mentioned in the theorem.

\section{BIBLIOGRAPHY}

1. A. A. Albert, Modern higher algebra, Chicago, 1937.

2. - Structure of algebras, Amer. Math. Soc. Colloquium Publications, vol. 24, New York, 1939.

3. N. Jacobson, Ann. of Math. vol. 38 (1937) p. 508.

4. - Ann. of Math. vol. 39 (1938) p. 181.

5. - Duke Math. J. vol. 3 (1938) p. 534.

6. P. Jordan, Nachr. Ges. Wiss. Göttingen (1932) p. 569.

7. - Nachr. Ges. Wiss. Göttingen (1933) p. 309.

8. Z Zeitschrift für Physik vol. 80 (1933) p. 285.

9. P. Jordan, J. von Neumann, and E. Wigner, Ann. of Math. vol. 35 (1934) p. 29.

10. W. Landherr, Abh. Math. Sem. Hamburgischen Univ. vol. 11 (1935) p. 41.

11. - Abh. Math. Sem. Hamburgischen Univ. vol. 12 (1938) p. 200.

\section{University of Chicago,} Chicago, Ill. 